\title{
Bericht \\ über neuere Untersuchungen und Probleme \\ aus der Theorie \\ der algebraischen Zahlkörper
}

Teil I: Klassenkörpertheorie

Teil Ia: Beweise zu Teil I

\author{
von \\ Dr. Helmut Hasse \\ o. Professor an der Universität Hamburg
}

2., durchgesehene Auflage

Springer-Verlag Berlin Heidelberg GmbH 1965 
Alle Rechte, insbesondere das der Obersetzung in fremde Sprachen, vorbehalten. Nachdruck und photomechanische Wiedergabe, auch von Teilen, nicht gestattet.

(C)

Springer-Verlag Berlin Heidelberg 1965

Ursprünglich erschienen bei Physica -Verlag Rudolf Liebing K. -G., Würzburg 1965 


\section{Teil I: Klassenkörpertheorie.}

Seite

§ 2. Die Hilbert-Furtwänglerschen Sätze über Klassenkörper. . . . . . . . 3

§ 3. Verallgemeinerung des Idealklassenbegriffs (Weber, Takagi). . . . . . 4

§ 4. Die Takagischen Sătze über Klassenkörper . . . . . . . . . . . . . 9

§ 5. Umgruppierung der zu beweisenden Tatsachen . . . . . . . . . . . 11

§ 6. Die eigentlichen Beweise der Takagischen Sätze. . . . . . . . . 16

§.7. Das Zerlegungsgesetz für die Teiler der Relativdiskriminante . . . . . 30

§ 8. Der Satz von der arithmetischen Progression in $k$. . . . . . . . . 31

§ 9. Die Heckesche Funktionalgleichung der $L$-Reihen . . . . . . . . . . 35

$\S$ 10. Die absolut-Abelschen Körper und die zu einem imaginär-quadratischen

Grundkörper Abelschen Körper. . . . . . . . . . . . . . . . . . . 39

§ 11. Drei ungelöste Probleme aus der Theorie der relativ-Abelschen Körper 44

Erläuterungen . . . . . . . . . . . . . . . . . . . 48

Berichtigungen und Ergänzangen ................56

Bem.: Die Zeichen *),*), ... beziehen sich auf die unter dem Text gebrachten Anmerkungen, die fortlaufenden Ziffern $\left.\left.{ }^{1}\right),{ }^{2}\right), \ldots$ dagegen auf die am SchluB des Berichtes angefügten Erläuterungen.

\section{Teil Ia: Beweise zu Teil $\mathbf{I}^{1}$ )}

Einleitung .......... ............. 58

§ 1. Hilfssätze über Abelsche Grappen . . . . . . . . . . . . . 58

\$ 2. Die unendlichen Primstellen. . . . . . . . . . . . . . 59

§ 3. Die additiven und multiplikativen Restklassengruppen. . . . . . 6 60

§4. Die Idealgruppen mod. $\tilde{\mathfrak{m}} \ldots . . . . . . . . . . . . .63$

§. Die Idealklassenzahl $h^{(\tilde{\mathrm{m}})}$ nach einer Hauptidealgruppe $H^{(\tilde{\mathfrak{m}})} \ldots \ldots 6$

§ 6. Zusätzliche Bemerkungen zu den Definitionen und Sätzen der Klassenkörpertheorie hinsichtlich der unendlichen Primstellen. . . . . 65

§ 7. Genaue Ausführung von I, § 6, B), Reduktion 2 . . . . . . . . 67

§ 8. Theorie des relativ-Galoisschen Körpers . . . . . . . . . . . 69

§9. Die Relativdiskriminante eines relativ-zyklischen Körpers von Primzahlgrad ................... 74

§ 10. Die primen Normenreste eines relativ-zyklischen Körpers von Primzahlgrad ................... 77

§ 11. Kummersche Körper $(0=1)$. . . . . . . . . . . . . . . . 86

§ 12. Das Einheitenhauptgeschlecht eines relativ-zyklischen Körpers von Primzahlgrad. . . . . . . . . . . . . . . . 91

1) Teil I wird kurz mit I zitiert. Verweise ohne diese Angabe beziehen sich auf den vorliegenden Teil Ia. 


\section{In h a l t.}

§ 13. Die ambigen Idealklassen eines relativ-zyklischen Körpers von Primzahlgrad . . . . . . . . . . . . . . . . . . . . . . 98

§ 14. Genaue Ausführung des Beweises von I, §6, Sătze $\left(B^{\prime \prime}\right),\left(B^{\prime \prime \prime}\right),\left(B^{\prime \prime \prime \prime}\right)$. 101

\$ 15. Dio Anzahl $t$ der verschiedenen Idealgruppen $H$ mod. $\tilde{f}$ vom Primzshlindex $l$ im Falle $o=1$. . . . . . . . . . . . . . . 103

§ 16. Die Anzahl $\bar{t}$ der verschiedenen Kummerschen Korper $K=k(\sqrt[b]{\mu})$, deren Modul $\tilde{f}_{K k}$ Teiler von $\tilde{f}$ ist $(0=1) \ldots . . . . .110$

§ 17. Genaue Ausführung des Beweises von I, § 6, Satz (B'). . . . . . . . 112

§ 18. Genaue Ausführung des Beweises von I, \& 6, B), Reduktion 3 . . . . 114

\$ 19. Der allgemeinste Hauptgeschlechtsatz für einen relativ-zyklischen Körper von Primzahlgrad . . . . . . . . . . . . . . . . . . 120

§ 20. Genaue Ausführung des Beweises von I, \& 6, Satz (C) . . . . . . . 128

§ 21. Genaue Ausführung zum Beweise von I, \& 6, Satz (D), 8 . . . . . . 134 Bezeichnungstabelle zu don \$8 9-19 als Tafel am SchluB. 